\title{
Dynamic plasma-wall modeling of ELMy H-mode with UEDGE-MB-W
}

\author{
A.Yu. Pigarov ${ }^{\text {a }}$, S.I. Krasheninnikov ${ }^{\mathrm{a}, \mathrm{b}}$, T.D. Rognlien ${ }^{\mathrm{c}}$, C.J. Lasnier ${ }^{\mathrm{c}}$, E. Unterberg ${ }^{\mathrm{d}}$ \\ ${ }^{a}$ University of California at San Diego, 9500 Gilman Dr., La Jolla, CA 92093-0417, USA \\ ${ }^{b}$ Nuclear Research National University, MEPhI, Moscow, 115409, Russia \\ ${ }^{c}$ Lawrence Livermore National Laboratory, Livermore, CA 94551, USA \\ ${ }^{d}$ Oak Ridge National Laboratory, Oak Ridge, TN 37831, USA
}

\begin{abstract}
The results of time-dependent coupled simulations of the boundary plasma and the first wall deuterium inventory in various sequences of many type-I ELMs with UEDGE-MB-W code are presented. The temporal evolution of deuterium inventories of the pedestal plasma and wall and the calculated rates of particle deposition into the wall during ELMs and of wall outgassing between ELMs are compared to the experimental data on DIII-D. The fraction of pedestal particle losses deposited into the wall during ELMs is studied for different sizes and frequencies of ELMs. Modeling results for the discharge exhibiting the transition from small to giant type-I ELMs due to NBI heating decrease are discussed, demonstrating the important role of wall outgassing in the pedestal density built-up. The dynamic deposition/release equilibrium attained in the saturated wall in a sequence of ELMs and the roles of different plasma-material interaction processes in generating gas release are analyzed.
\end{abstract}

PACS: $52.55 . \mathrm{Fa}, 52.65 . \mathrm{Kj}$

PSI-20 keywords: Edge plasma, ELM, Intermittent transport, DIII-D, dynamic retention *Corresponding author address:

*Corresponding author e-mail: apigarov@ucsd.edu

Presenting author: A.Yu. Pigarov Presenting author e-mail: apigarov@ucsd.edu 


\section{Introduction}

The boundary plasma in magnetic fusion devices is breathing due to repetitive transient events known as Edge Localized Modes (ELMs). In these events, large amounts of plasma energy and particles are expelled from the confined plasma region onto material surfaces during a very short time interval. Since the expelled particles are transiently deposited into material surfaces and then gradually released back to the plasma on a much longer inter-ELM time scale, the boundary plasma and wall can be thought of as breathing together [1]. In this case, particle inventories of the boundary plasma and the first wall are strongly coupled and should be modeled self-consistently. In the paper we present the results of time-dependent modeling of ELMy H-mode plasmas with the UEDGE-MB-W code.

\section{UEDGE-MB-W model}

The 2-D multi-fluid transport code UEDGE has been recently extended [1]. The new multiphysics version UEDGE-MB-W incorporates the Macro-Blob approach [1-3] for intermittent non-diffusive transport due to filamentary coherent structures observed during ELMs and simple built-in models for simulating the onset of the pedestal transport barrier and the timedependent recycling, surface temperature, and wall gas inventory for each material boundary of the 2-D modeling domain. In the Macro-Blob approach, the numerous filaments are combined into a series of continuous toroidally-symmetric structures called macro-blobs (MBs). The MBs are generated in the pedestal region, localized at the outboard part of the torus (in consistency with the coupled peeling-ballooning instability [1]), and ballistically advected on a 2-D curvilinear mesh in the cross-field direction. In the paper, we model MBs of about $\Delta_{\mathrm{mb}}=0.1 \mathrm{~m}$ in radial size and $2.3 \mathrm{~m}^{3}$ in volume propagating at the velocity of $1 \mathrm{~km} / \mathrm{s}$. The ELM event is simulated as a sequence of MBs, in which the number of MBs, $\mathrm{N}_{\mathrm{mb}}$, is an 
adjustable parameter to match the experimental data on pedestal particle/energy losses. For each material boundary cell of the modeling domain, UEDGE-MB-W solves: (i) 1-D heat conduction equation to find the surface temperature $T_{\mathrm{w}}(t)$, and (ii) $0-\mathrm{D}$ equation for deuterium inventory $N_{\mathrm{dw}}(t)$ in the material, $d N_{\mathrm{dw}} / d t=\Phi_{\mathrm{p}}(t)[1-R(t)]-S_{\text {therm }}(t)-S_{\mathrm{sd}}(t)$. Here $\Phi_{\mathrm{p}}$ is the incident plasma flux, $R=R_{\mathrm{elm}} \mathrm{Y}(t)+R_{\mathrm{st}}[1-\mathrm{Y}(\mathrm{t})]$ is the recycling coefficient, $S_{\mathrm{therm}}=S_{\mathrm{th}} F_{\mathrm{th}} \varphi\left(\Phi_{\mathrm{p}}\right) \mathrm{Y}$ and

$S_{\mathrm{sd}}=S_{\mathrm{w}} \varphi\left(\Phi_{\mathrm{p}}\right)[1-\mathrm{Y}]$ are the wall outgassing rates due to thermal and ion-stimulated desorption, functions $F_{\mathrm{th}}=\delta\left(T_{\mathrm{w}}(t)>T_{\mathrm{a}}\right), T_{\mathrm{\alpha}} \sim 500 \mathrm{C}$ for graphite, $\varphi$ characterizes the deposition profile over the first wall. When MBs interact with a given material surface, $\mathrm{Y}=1$, and $\mathrm{Y}=0$, otherwise. $S_{\mathrm{th}}$ and $S_{\mathrm{w}}$ are the source strength values. In the paper, analysis will be given to the total inventory, which is a sum over all material cells.

\section{Dynamic equilibrium in ELMy plasma}

With UEDGE-MB-W we model the periodic sequences of ELMs (Fig. 1, top) considering the ELM period $\left(\mathrm{T}_{\mathrm{elm}}\right)$ as an input parameter. In calculations, we take $\mathrm{N}_{\mathrm{mb}}=1, R_{\mathrm{elm}}=0.8, S_{\mathrm{th}}=0$, $S_{\mathrm{w}}=12.5$ Torr-1/s, profiles of transport coefficients given in [1], no external gas puff and start from the same initial plasma state. The dependence of boundary plasma particle inventory taken at the end of ELM cycle on the number of ELMs for different $T_{\text {elm }}$ is shown in Fig. 1, middle. As can be seen, the sequences converge to a dynamic equilibrium (DE), in which the plasma (and the first wall) parameters averaged over the ELM cycle practically do not vary with increasing number of ELMs. Characteristic times of reaching the dynamic equilibrium are long, about a second. The plasma line densities (integrated along the pedestal interferometer chord) in the DE are shown in Fig. 1, bottom, as a function of $\mathrm{T}_{\text {elm. }}$. As seen, the plasma density drops while $\mathrm{T}_{\text {elm }}$ decreases. In the case of frequent ELMs, $\mathrm{T}_{\text {elm }} \leq 20 \mathrm{~ms}$, the time-average radial plasma transport caused by ELMs is very strong and the resulting DE 
density is much smaller than that for $\mathrm{T}_{\text {elm }}>30 \mathrm{~ms}$. This trend is in agreement with the measured densities in DIII-D shots with different NBI power and without gas puff. Note, strong external gas puff $\geq 100$ Torr- $1 / \mathrm{s}$ into H-mode on DIII-D is required to maintain the plasma density at high level in the case of frequent ELMs.

\section{Time-dependent recycling in ELMy H-mode}

We use UEDGE-MB-W to simulate experimental data for the shot \#144977 on DIII-D with giant type-I ELMs. We focus on two consecutive ELM events (Fig. 2) at times $\tau_{\text {elm } 1}=2.9225$ and $\tau_{\text {elm2 }}=3.0725 \mathrm{~s}$, which result in different pedestal plasma particle losses of $\sim 25 \%$ and $\sim 20 \%$ (according to both interferometry and volume integration Thomson Scattering System (TSS) data) and differ in their duration from $\sim 2.5$ to $\sim 1.6 \mathrm{~ms}$. The pedestal particle and energy inventories at the beginning of the first ELM are almost completely restored during $\sim 150 \mathrm{~ms}$ before the second ELM. The main fitting parameters of our model are $\mathrm{N}_{\mathrm{mb}}, R_{\mathrm{elm}}, S_{\mathrm{th}}$ and $S_{\mathrm{w}}$ and transport coefficients with dynamic onset of transport barrier. In a series of runs modeling sequences of several ELMs, we adjust the fitting parameters to match the temporal evolution of the boundary plasma [1]. Our wall model simulates only the dynamic part of deuterium inventory, which can be directly compared to the experimental inventory obtained from time-dependent analysis of the discharge gas balance. Since we are not simulating the whole discharge, the calculated inventory is normalized to its experimental value at the beginning of the first ELM. The best fit corresponds to $\mathrm{N}_{\mathrm{mb}}=4$ and 3 for these ELM events, and $R_{\mathrm{elm}}=0.8, S_{\mathrm{th}}=0, S_{\mathrm{w}}=12.5$ Torr-1/s. In Fig. 2 , top panel, the calculated temporal evolution of pedestal $\mathrm{D}^{+}$inventory is compared to the experimental one obtained from TSS data (extracting the impurity contibution based on CER measurements). The calculated and experimental temporal evolutions of deuterium wall inventory are shown in the bottom panel. 
As seen, the plasma and wall inventories vary synchronously. During ELMs, the wall is in the state of net particle deposition but switches to the net outgassing between ELMs. The wall inventories decrease between ELMs practically linearly, in agreement with UEDGE-MB-W assumption of constant $S_{\mathrm{w}}$, as well as experimental gas balance. The value of $S_{\mathrm{w}} \approx 8 \times 10^{21} \mathrm{D} / \mathrm{s}$, which is also in agreement with experimental wall outgassing rate [1], is much larger than the NBI fueling and pumping rates.

\section{Role of wall in ELMy H-mode}

The UEDGE-MB-W simulations matching the experimental data on temporal evolution of pedestal plasma parameters and deuterium wall inventory during the ELM cycle are performed for a few DIII-D shots (type-I ELMs, NBI heating, and no gas puff into H-mode) with different ELM periods: 20, 45, 125 and 150 ms. In modeling, we vary the number and initial volume of macro-blobs, whereas the recycling coefficient and outgassing rate have roughly the same values $R_{\mathrm{elm}}=0.8$ and $S_{\mathrm{w}}=12.5$ Torr- $1 / \mathrm{s}$. The calculated fraction of the pedestal particle losses deposited into the wall during an ELM, $\zeta_{\text {wall, }}$ is shown on Fig. 3, top panel, as a function of the ELM period. As seen, the higher the ELM period (and, correspondingly, the higher pedestal density), the larger the fraction of particles deposited into the wall during ELMs is. In the case of giant infrequent ELMs $\left(\mathrm{T}_{\text {elm }}>100 \mathrm{~ms}\right)$, the deposition into the wall dominates, as $\zeta_{\text {wall }}$ is up to $80 \%$. In dynamic equilibrium, the wall outgassing rate between ELMs is high enough to compensate for the pedestal plasma particle losses during the ELM. For frequent ELMs $\left(\mathrm{T}_{\mathrm{elm}}<30 \mathrm{~ms}\right), \zeta_{\text {wall }}$ is much smaller, $\sim 20 \%$, as more particles after the ELM are retained in the edge plasma regions (SOL, private flux, and divertors).

With UEDGE-MB-W we also simulate a larger variety of ELM sequences, all reaching a dynamic equilibrium. These sequences differ in the size and frequency of ELMs 
and in the divertor inter-ELM plasma parameters, whereas the magnetic configuration, input power and wall outgassing rate are the same. We find that the faction $\zeta_{\text {wall }}$ depends strongly on the ELM size characterized by the pedestal particle loss $\left(\mathrm{N}_{\text {pedlos }}\right)$ as well as on the total particle inventory in the edge regions before ELMs $\left(\mathrm{N}_{\text {soldiv }}\right)$. Giant ELMs are typically longer in duration and their modeling requires several macro-blobs. The divertor recycling strongly increases with $\mathrm{N}_{\mathrm{mb}}$, thus significantly increases $\zeta_{\text {wall }}$ as demonstrated by the following simple estimates. The pedestal, edge, and wall inventories vary with macro-blob index $k$ as $g_{p, k}=g_{p, k}$ I( $(1-p), g_{e, k}=\gamma_{e d, k}(1-r), g_{w, k}=r \Sigma_{1 \leq i \leq k} \gamma_{e d, k}$, where $\gamma_{e d, k}=g_{e, k-1}+g_{p, k-1} p, g_{p, 0}=1, g_{e, 0}=a$, coefficients $p$ and $r$ denote the fractions of pedestal and edge losses per macro-blob, and $\zeta_{\text {wall }}\left(\mathrm{N}_{\mathrm{mb}}\right)=g_{w, k} /(1-$ $\left.g_{p, k}\right), k=\mathrm{N}_{\mathrm{mb}}$. For small ELMs, $\mathrm{N}_{\mathrm{mb}}=1, p / a \leq 2, r \leq 1-R_{\mathrm{elm}}=0.2$, thus $\zeta_{\mathrm{wall}}=r(p+a) / p \sim 1-R_{\mathrm{elm}} \approx 0.2$. For giant ELMs, $\mathrm{N}_{\mathrm{mb}}=5,\left(1-g_{p, 4}\right) / a \sim 4, r \sim 1-R_{\mathrm{elm}}=0.2$, we obtain large $\zeta_{\text {wall }} \sim 0.65$ in agreement with UEDGE-MB-W modeling.

As a figure of merit in comparative analysis of various ELM sequences modeled with UEDGE-MB-W, we introduce the parameter $\eta=\mathrm{N}_{\text {pedlos }} / \mathrm{N}_{\text {soldiv }}$. The main trend is demonstrated in the chart on Fig. 3, bottom panel, where we show the fractions of pedestal particle loss transiently retained in the wall and edge regions after an ELM for different values of $\eta$. As seen, in the case $\eta \geq 4$, in which the number of particles expelled from the pedestal during the ELM is large compared to the initial edge particle inventory, the dominant fraction $\sim 70 \%$ is deposited into the wall. The pedestal recovery after the ELM is determined mainly by the wall physics processes assocated with wall outgassing. This case corresponds to giant infrequent ELMs. In the oposite case, $\eta \leq 2$, which corresponds to small type-I and type-III ELMs, the dominant fraction of expelled particles is transiently retained in the edge regions. In this case, the pedestal recovery is determined by the physics of edge plasma, e.g., by the 
leakage of neutrals from the divertors, anomalous inward pinch in the pedestal transport barrier region, and etc.

\section{Wall-related processes}

A sequence of many identical ELMs results in the dynamic deposition/release equilibrium (DDRE) for deuterium in the first wall. For analysis of DDRE properities, we use the following 0-D model, which distinguishes two overlaping regions inside the ELM-affected material surfaces: $0 \leq z \leq L_{1}$ (region 1) and $0 \leq z \leq L_{2}$ (region 2). These regions are responsible for plasma-wall interactions between and during ELMs, respectively. Temporal evolution of deuterium densities in these regions is given by equations: $L_{1} d n_{1} / d t=\left(1-\mathrm{r}_{1}\right) \Phi_{1}-\gamma_{1} \Phi_{1} n_{1} / \mathrm{n}_{\mathrm{ds}}$ for $t_{\mathrm{elm}}<t<\mathrm{T}_{\mathrm{elm}}$, and $L_{2} d n_{2} / d t=\left(1-\mathrm{r}_{2}\right) \Phi_{2}-\gamma_{2} \Phi_{2} n_{2} / \mathrm{n}_{\mathrm{ds}}$ for $0<t<t_{\mathrm{elm}}$, where $L, \Phi, \mathrm{r}$, and $\gamma$ denote the implantation depth, incident plasma flux, backscattering coefficient and ion-stimulated desorption coefficient, respectively. Equations are coupled via periodicity conditions before and after the ELM: $n_{2}(0)=n_{1}\left(\mathrm{~T}_{\text {elm }}\right), n_{1}\left(\mathrm{t}_{\text {elm }}\right)=n_{2}\left(\mathrm{t}_{\text {elm }}\right)$. The first terms in the $r h s$ describe implantation of particles and the second terms stand for desorption. For simplicity, we neglect long time scale terms, like deuterium diffusion into the material bulk, responsible for net wall pumping over the ELM cycle. The characteristic radial widths of particle deposition and release are $\Delta_{\mathrm{mb}}$ and $\Delta_{1}$. The solution is: $n_{1}=\mathrm{g}_{1}-\left(\mathrm{g}_{1}-\mathrm{g}_{2}\right) \varepsilon_{1} \exp \left(-\mathrm{v}_{1} t\right), n_{2}=\mathrm{g}_{2}-\left(\mathrm{g}_{2}-\mathrm{g}_{1}\right) \varepsilon_{2} \exp \left(-\mathrm{v}_{2} t\right)$, where $v=\gamma \Phi /\left(\mathrm{n}_{\mathrm{ds}} L\right), \mathrm{g}=\mathrm{n}_{\mathrm{ds}}(1-\mathrm{r}) / \gamma, \varepsilon_{1}=\left[1-\exp \left(-\mathrm{v}_{2} t_{\mathrm{elm}}\right)\right] / h, \varepsilon_{2}=\left[1-\exp \left(-\mathrm{v}_{1}\left(\mathrm{~T}_{\mathrm{elm}}-t_{\mathrm{elm}}\right)\right] / h, h=1-\exp (-\right.$ $\left.v_{2} t_{\mathrm{elm}}\right) \exp \left[\left(-\mathrm{v}_{1}\left(\mathrm{~T}_{\mathrm{elm}}-t_{\mathrm{elm}}\right)\right]\right.$. For plasma conditions of giant ELMs simulated with UEDGE-MB$\mathrm{W}$, we evaluate $L_{1}=17 \mathrm{~nm}, L_{2}=40 \mathrm{~nm}, \mathrm{r}_{1}=0.5$, and $\mathrm{r}_{2}=0.01$ based on TRIM data for graphite, and take $\Phi_{1}=\Phi_{\mathrm{p}} \Delta_{1} / \Delta_{\mathrm{mb}}, \quad \Phi_{\mathrm{p}}=10^{19}, \quad \Phi_{2}=10^{20} \mathrm{~cm}^{-2} \mathrm{~s}^{-1}, \quad t_{\mathrm{elm}}=1.5 \mathrm{~ms}, \quad \mathrm{~T}_{\mathrm{elm}}=0.15 \mathrm{~s}, \quad \Delta_{1}=2 \mathrm{~cm}$. Parameters $\gamma_{1}, \gamma_{2}$, and $\mathrm{n}_{\mathrm{ds}}$ are unknown and their values can be deduced from matching the $R_{\mathrm{elm}}=\mathrm{r}_{2}+\gamma_{2}\left\langle n_{2}\right\rangle, \quad \mathrm{Y}_{\mathrm{ogs}}=\mathrm{r}_{1}+\gamma_{1}\left\langle n_{1}\right\rangle-1$, and $S_{\mathrm{w}}=\mathrm{Y}_{\mathrm{ogs}} \Phi_{1} 2 \pi \mathrm{R}_{\mathrm{p}} \Delta_{\mathrm{mb}}$. In Section 4 , we found that 
$R_{\text {elm }}=0.8-0.85$ and $S_{\mathrm{w}}=10-15$ Torr-1/s. We also determined from modeling the outgassing yield as $\mathrm{Y}_{\mathrm{ogs}}=S_{\mathrm{w}} / \Gamma_{\mathrm{p}}=0.5-1 \%$, where $\Gamma_{\mathrm{p}}$ is deuterium plasma flux on divertor plates. The best fit values are: $\gamma_{1} \approx 0.04, \gamma_{2} \approx 0.06$, and $\mathrm{n}_{\mathrm{ds}} \approx 8 \times 10^{21} \mathrm{~cm}^{-3}$. Three important conclusions can be made from the analysis of the obtained solution. First, the corresponding DDRE is characterized by the time average densities $\left\langle n_{1} \curvearrowright \approx 9.86 \times 10^{22} \mathrm{~cm}^{-3},\left\langle n_{2}\right\rangle \approx 9.92 \times 10^{22} \mathrm{~cm}^{-3}\right.$, which are close to the graphite density $10^{23} \mathrm{~cm}^{-3}$. The relative variation of inventory during the ELM cycle does not exceed $7 \%$. Even small values of desorption coefficients result in large outgassing fluxes. Such conditions are consistent with the supersatured layer concept discussed and modeled with WALLPSI code [4]. Second, the outgassing rate between ELMs is roughly constant, because $v_{1}\left(T_{\mathrm{elm}}-t_{\mathrm{elm}}\right)<<1$. Finally, the deduced $\gamma \sim 0.05$ is in good agreement with typical value of chemical erosion yield, $\sim 1 \%$, when deuterium is released in the form of methane.

\section{Transition from frequent to infrequent ELMs}

The important role of the first wall in the plasma performance is also highlighted in the DIIID shot \#148980 exhibiting a transition from the small and frequent $\left(\mathrm{T}_{\text {elm }} \approx 20 \mathrm{~ms}\right)$ to the giant and infrequent $\left(\mathrm{T}_{\text {elm }} \approx 50 \mathrm{~ms}\right)$ ELMs due to decrease in the NBI heating power from 3 to 2.5MW (Fig. 4A). The transition results in substantial increase of plasma density from $4.3 \times 10^{19}$ to $5.1 \times 10^{19} \mathrm{~m}^{-3}$. As there is no external gas puff during the H-mode phase, the wall is the only source of particles necessary for the pedestal built-up. The decrease in deuterium wall inventory for $\sim 0.5 \mathrm{~s}$ followed the transition is confirmed by the experimental gas balance analysis (Fig. 4B). Without this transition the wall inventory is expected to increase gradually, because the wall is the area of net deposition over the whole ELM cycle with the net pumping rate about the NBI fueling rate $S_{n b i}$. The deduced experimental rate of wall outgassing between ELMs over the H-mode phase practically remains at the same constant 
level $\sim 13$ Torr-1/s (Fig. 4D). Deposition rates during ELMs before and after the transition are different (Fig. 4C). The net deuterium deposition during giant infrequent ELMs is by a factor of 2 higher than that during frequent ELMs. In UEDGE-MB-W modeling of this shot, we first obtain the dynamic plasma equilibrium with frequent ELMs and, then, change the ELM period and NBI power at transition time. The calculated evolution of pedestal line density is shown on Fig. 4E. As seen, the characteristic time of density built-up is about $0.5 \mathrm{~s}$ in agreement with experimental data. The plasma density increases because more particles release from the wall during the longer ELM cycle. It is likely that without strong wall outgassing, the characteristics of ELMs will be different.

\section{Concluding remarks}

The UEDGE-MB-W code simulations were performed for NBI-fueled H-mode plasmas obtained in the DIII-D tokamak with different plasma densities and ELM frequencies. In a periodic sequence of many identical ELMs, the simulated particle inventories of the boundary plasma and the first wall simultaneously evolve to a dynamic equilibrium, in which their values averaged over the ELM cycle practically do not vary from one ELM to the next. During a single ELM, the wall is in the state of net particle deposition but switches to the net outgassing between ELMs. In the case of the giant infrequent ELMs observed on DIII-D, the amount of particles expelled from the pedestal plasma is much larger than the particle inventory in the edge plasma regions before the ELM. In this case, the expelled particles are predominantly deposited into the wall and the recovery of the pedestal plasma after the ELM is determined by the wall physics processes. It was found that the wall outgassing rate between giant type-I ELMs is roughly constant and its magnitude is high enough to compensate for the pedestal particle losses during an ELM. This rate is much larger than the 
NBI fueling and pumping rates. It is expected that the so-called ion-stimulated desorption from the supersaturated layers is the dominant process of graphite wall outgassing between ELMs. Modeling of the temporal evolution of the plasma discharge featuring the transition from the small and frequent to the large and infrequent ELMs shows that the observed increase in the discharge density corresponds to the decrease in the particle inventory of the wall and the characteric time of transition is consistent with the deduced wall outgassing rate. The coupled evolution of the boundary plasma and material wall is very important because it implies that the physics of wall loading and outgassing has strong impact on the fusion plasma performance. The temporal evolution of boundary plasma parameters and the characteristics of transient events in long-pulse next-step fusion devices can be greatly influenced by the wall physics and the choice of wall materials.

This work performed under the auspices of the U.S. Department of Energy under DEFG02-04ER54739, DE-AC52-07NA27344, and DE-AC05-00OR22725.

\section{References}

[1] A.Yu. Pigarov, et al., Phys. Plasmas 21, 062514 (2014).

[2] A.Yu. Pigarov, et al., Phys. Plasmas 19, 072516 (2012).

[3] A.Yu. Pigarov, et al., Phys. Plasmas 18, 092503 (2011).

[4] A.Yu. Pigarov, et al., Contributions Plasma Physics 52, 465 (2012).

\section{Figure captions}

Fig.1: Periodic sequence of identical ELMs is shown schematically in the top panel. Variation of boundary plasma inventory with the number of ELMs in a sequence (middle). Pedestal density integrated along the boundary interferometer chord as a function of $\mathrm{T}_{\text {elm }}$ 
(bottom). Experimental data representing the NBI-power scan on DIII-D are shown by squares.

Fig.2: The calculated (lines) and experimental (points) data on temporal evolution of deuterium inventories of the boundary plasma (top) and the first wall (bottom) are compared.

Fig.3: Fraction $\zeta_{\text {wall }}$ as a function of ELM period (top). Fractions of pedestal particle loss retained in the wall and in the edge plasma for different values of parameter $\eta$ (bottom).

Fig.4: Temporal evolution of plasma density (A), dynamic wall inventory (B), deuterium deposition (C) and release (D) rates for the discharge with transition from frequent to infrequent ELMs at time $2.85 \mathrm{~s}$. Boundary line densities calculated with UEDGE-MB-W (solid curve is connecting the densities attained at the end of ELM cycle) and measured by the boundary interferometer at times just before ELMs (points) are displayed on the panel (E). 
Fig.1:

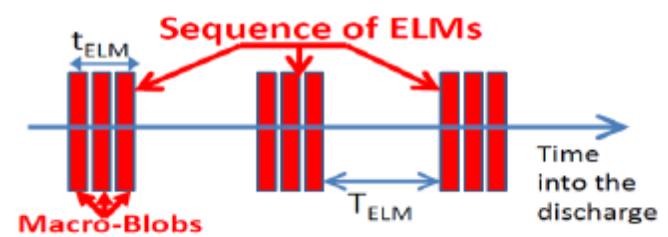

Boundary particle inventory,Torr-L/s

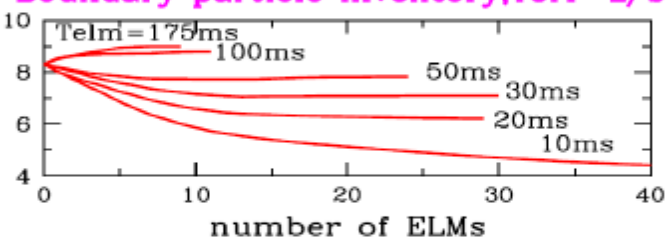

Boundary line density, $10^{14} \mathrm{~cm}^{-3} / \mathrm{m}$

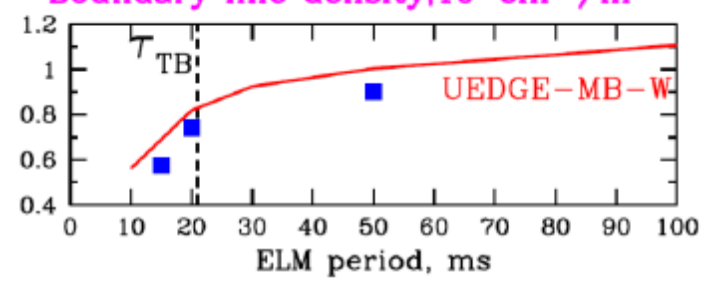

Fig.2

Deuterium plasma inventory, $10^{20}$



Deuterium wall inventory, $10^{20}$

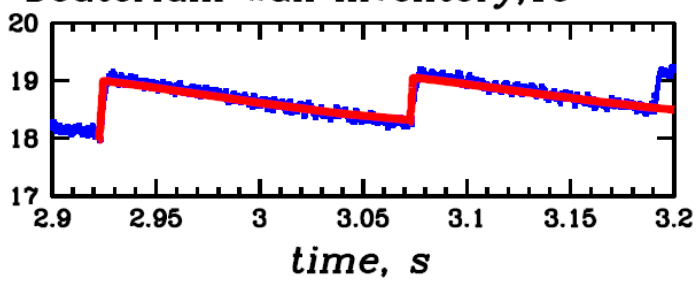

Fig.3 

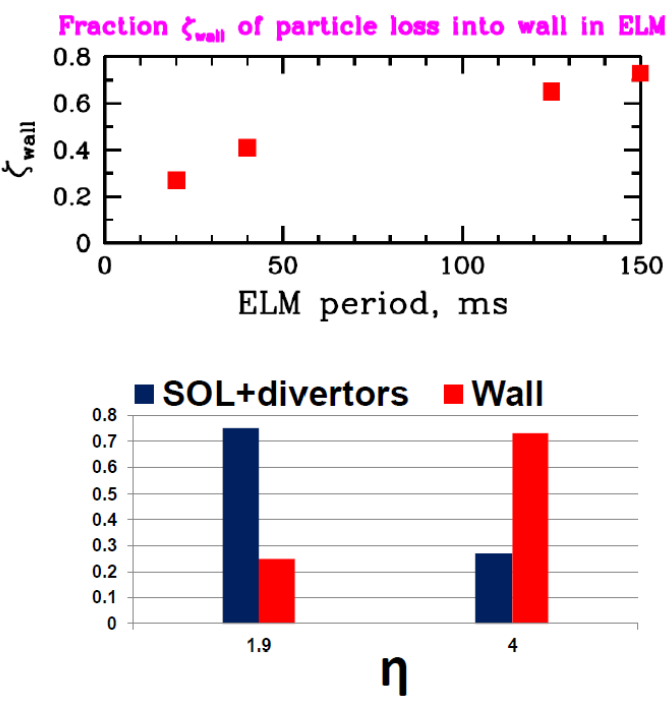

Fig.4

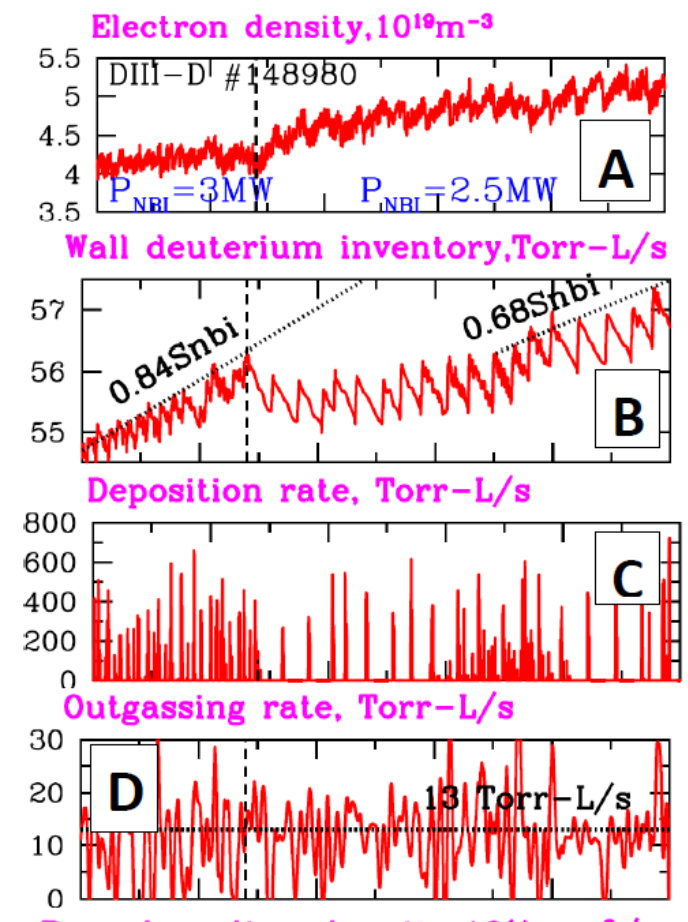

Boundary line density, $10^{14} \mathrm{~cm}^{-3} / \mathrm{m}$

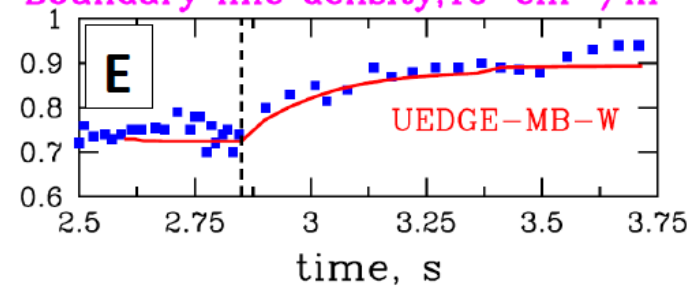

